\title{
A Combined Localization-Synchronization Method for Underwater Communication
}

\author{
ZAHRA MOUSAVI $^{1}$ and REZA JAVIDAN ${ }^{2}$ \\ ${ }^{1,2}$ Department of Computer Engineering and Information Technology, Shiraz University of Technology \\ E-mail: 'zmousavi1386@gmail.com,r2reza.javidan@gmail.com
}

\begin{abstract}
In underwater communication usually localization and time synchronization of nodes are important processes which are treated separately. However in Wireless Sensor Networks (UWSNs) saving energy of nodes is another important factor. To save energy, it is an increasing interest to do these works together. In this paper a new method for combined localization and synchronization for communications in UWSNs is proposed. In this method the attribute of sound wave has been considered and stratification effect is used to compensate the bias in range estimates. This is a main important factor that is not considered in other related researches. In addition, mobility of nodes is considered as another parameter in this solution. Simulation results on prototype data showed the effectiveness of the proposed method. In addition, it is compared with another well-known method and the results show the outperforming results for the proposed method.
\end{abstract}

Keywords: Synchronization, Localization, Stratification effect, Underwater Wireless Sensor Network.

\section{INTRODUCTION}

In most underwater communications, localization and clock synchronization are two key elements. Especially in most Wireless Sensor Network (WSN) applications, it is a need for these two services. For instance, TDMA (Time Division Multiple Access), one of the commonly used Medium Access Control (MAC) protocols, often requires precise time synchronization between sensor nodes. Furthermore, most geographic routing algorithms assume the availability of location information [1], [2].

As another example, ranging in WSNs is usually performed by measuring the Time of Arrival (ToA), Time Difference of Arrival (TDoA), Received Signal Strength Indicator (RSSI), or Angle of Arrival (AoA) of received signals. However, for Underwater WSNs (UWSNs) an accurate attenuation model for the underwater acoustic channel is not available, and AoA techniques require multiple hydrophones. As a result, most approaches for UWSNs rely on ToA or TDoA for distance estimation [3] which causes that many localization algorithms in UWSNs rely on the time synchronization services. For instance, in TOA, synchronization is a prerequisite. On the other side, knowledge of location helps synchronization because it can be used to estimate propagation delays. Based on these bonds relationships between synchronization and localization, it is evident to investigate the possibility of formulating them into a unified framework.

Most previous researches assumed that the sound speed in underwater communication is constant in a known environment. However, In UWSNs, sound speed varieties with depth, called "stratification effect" and the real transmission path usually bends based on the range

In this paper a novel sequential approach for joint localization and synchronization in UWSNs is proposed that considers stratification effect. This approach which is based on packet exchanges between anchor and other nodes uses directional navigation systems employed in nodes to obtain accurate short-term motion estimates and taps the permanent motion of nodes. The proposed approach also allows self-evaluation of the localization accuracy that is sound speed is adjusted based on 
depth, temperature, and salinity in sea environment. Simulation results on prototype data showed the effectiveness of the proposed method.

The remainder of the paper is organized as follows: in Section 2, a review on related works for localization and synchronization are summarized. In Section 3 the proposed method is described. Simulation results are presented and discussed in Section 4. Finally, conclusions are outlined in Section 5.

\section{ARTICLE REVIEW}

There are many researches carried out for underwater acoustic localization. For instance Cheng [4] and Tan [5]-[6] suggested obtaining TDoA measurements for ranging by sequentially generating packets from anchor nodes when the anchor-to-anchor distance is known. It allows the ordinary node to remain silent and it is not required either the ordinary node or the anchor nodes to be time-synchronized. However, because of unpredictable MAC delays, an anchor node might not be able to respond immediately, hence significantly decreasing the accuracy of TDoA measurements. In these situations, ToA-based localization seems more suitable. ToA-based methods tend to be noisy due to synchronization errors and multipath. Assuming the availability of multiple ToA measurements at a static ordinary node, Tan at [6] uses Least Squares (LS) estimators to mitigate such noise. But this makes the system even more sensitive to node movements.

Mirza [7] proposed another method that compensate node movements, either by regarding these movements as ToA noise or by using mobility prediction. This approach considered node movements as an undesired phenomenon and do not utilize additional ToA measurements when coupled with self-localization.

One of the challenges of UWSNs is the variability of the propagation speed in water, Considering this problem, Kim and et al [8] suggested the first estimation for the propagation speed using packet exchange between floating buoys on the seabed and the sea surface. Furthermore Isik at [9] suggested propagation speed estimation based on measuring the channel characteristics and a sound speed model.

More recently methods considered synchronization and localization together. Tian and others [10], proposed the first localization and synchronization scheme for 3D underwater acoustic networks using atomic multilateration and iterative multilateration techniques. In this scheme, anchor nodes localized on the surface of the water and they send time and location information to other sensors. The drawback of this scheme is that it ignores the clock skew, which will lead to frequent and redundant synchronization.

Liu et al at [11] proposed iterative solution for joint localization and synchronization for UWSNs, called JSL. JSL is a four phases scheme in which time synchronization and localization are performed at different phases, and during iterations, the output of synchronization is fed back as the input of localization, and the output of localization is fed back as the input of synchronization. In JSL, stratification effect of underwater medium is considered and compensated. Additionally JSL assumes that anchor nodes are synchronized the other nodes are not. Finally advanced tracking algorithm IMM (interactive multiple model) is adopted to recuperate the accuracy of localization in the mobile case.

Diamant and Lampe at [12] described a sequential algorithm for joint time-synchronization and localization for underwater networks called STSL. It uses a two-step approach, i.e. at first nodes are synchronized and then location of them are estimated. This solution allows self-evaluation of the localization accuracy and considers mobility of nodes. JSL assumes that anchor nodes are timesynchronized but STSL assumes that anchor nodes are not time-synchronized. Drawback of this algorithm is that stratification effect is not considered and assumes that sound waves propagate in straight lines in water. In addition, multipath is not considered in STSL.

\section{THE PROPOSED METHOD}

In this paper a new method for combined localization and synchronization for communications in UWSNs is proposed. In this section the details of the proposed method are described. Notations that are used in this paper are explained in Table 1 which is included in Appendix 1. The procedure of the proposed method consists of five phases that are shown in Figure 1. The objective of the first to the last phases is to provide an estimation of the propagation delays $\mathrm{T}^{\mathrm{pd}}{ }_{\mathrm{i}}$, for any $\mathrm{i}$ $\epsilon N$. This is accomplished by a two-way packet exchange scheme but due to permanent movement of nodes, propagation delays $\mathrm{T}^{\mathrm{pd}}{ }_{\mathrm{i}}, \mathrm{i} \in \mathrm{N}^{\mathrm{r}}$ and $\mathrm{T}^{\mathrm{pd}}{ }_{\mathrm{j}}$, $\mathrm{j} \epsilon \mathrm{N}^{\mathrm{s}}$ might not be equal.

Many synchronization approaches for UWSNs deal with this problem by letting the receiving node respond as the limitation of any possible movements (e.g., [13]). In the proposed method in 
our paper quantization mechanism that proposed in $[12]$ is used for differences in the propagation delay of separate packets.

In the following, system organization and assumptions and phases of the proposed method are explained. Each of the five steps in the work follow of Figure 1 will be explained in a separate subsection.

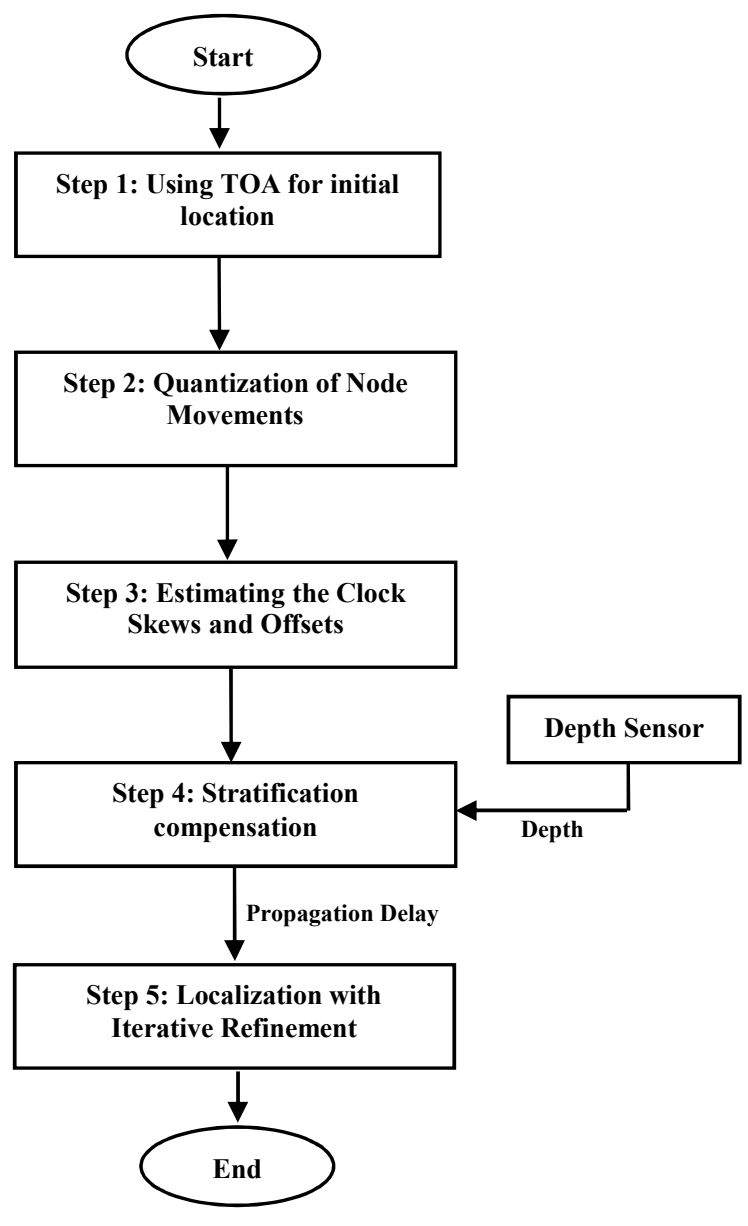

Fig. 1. The Workflow of proposed method

\subsection{System Organization and Assumptions}

Setting up of the assumptions includes that one or more ordinary nodes directly connected to $\mathrm{L} \geq 1$ anchor nodes and both ordinary and anchor nodes operate in a time-slotted UWSN. In addition, ordinary nodes perform localization independently of each other.

In this work, after a predefined localization window of duration as time slots, $\mathrm{W}$, we start with the ordinary node local time $\mathrm{t}^{\mathrm{O}}=0$, and then the $2 \mathrm{D}$ location of the ordinary node with $\mathrm{j}_{\mathrm{N}}=\left[\mathrm{j}_{\mathrm{N}}^{\mathrm{x}}, \mathrm{j}_{\mathrm{N}}^{\mathrm{y}}\right]^{\mathrm{T}}$ will be estimated.

It is assumed that nodes are not synchronized and the synchronization error is small relative to the globally established time-slot duration, such that a node can match a received packet with the time slot it was transmitted in. Therefore, local transmission times of received packets are known. The local clock of ordinary node is computed according to the Equation (1).

$$
\mathrm{t}^{\mathrm{O}}=\mathrm{t}_{\ell} \cdot \mathrm{S}_{\ell}+\mathrm{O}_{\ell}
$$

For localization, ordinary nodes and the anchors exchange packet based on two-way packet exchanges [14], so that packet $i \in \mathrm{N}^{\mathrm{r}}\left(\mathrm{N}^{\mathrm{r}} \in \mathrm{N}\right.$, is the number of packets that send in duration of localization window $\mathrm{W}$ ) transmit at local time $\mathrm{T}_{\mathrm{i}}$ and will detect by the ordinary node at anchor node $\ell_{i}$ local time $T_{i}+T^{p d}{ }_{i}+\gamma_{i}\left(\gamma_{i}\right.$ is a propagation delay measurement noise sample and it is zero-mean i.i.d. Gaussian random variable with variance $\sigma^{2}$ ), on the other side, packet $i \in \mathrm{N}^{\mathrm{S}}\left(\mathrm{N}^{\mathrm{S}} \in \mathrm{N}\right.$, is the number of packets that send from ordinary node in duration of localization window, $\mathrm{N}^{\mathrm{r}} \mathrm{U} \mathrm{N}^{\mathrm{s}}=\mathrm{N}$ ) receive by anchor node $l_{i}$ at local time $R_{i}+\gamma_{i}$ and transmit at anchor node $\ell_{\mathrm{i}}$ local time $\mathrm{R}_{\mathrm{i}}+\gamma_{\mathrm{i}}-\mathrm{T}^{\mathrm{pd}}$. Therefore, according to Equation (1) the following two equations will be deduced

$$
\begin{aligned}
& \mathrm{R}_{\mathrm{i}=} \mathrm{S}_{\ell_{\mathrm{i}}}\left(\mathrm{T}_{\mathrm{i}}+\mathrm{T}^{\mathrm{pd}}{ }_{\mathrm{i}}+\gamma_{\mathrm{i}}\right)+\mathrm{O}_{\ell_{\mathrm{i}}} \quad \mathrm{i} \in \mathrm{N}^{\mathrm{r}} \\
& \mathrm{T}_{\mathrm{i}=} \mathrm{S}_{\ell_{\mathrm{i}}}\left(\mathrm{R}_{\mathrm{i}}+\gamma_{\mathrm{i}}-\mathrm{T}_{\mathrm{i}}^{\mathrm{pd}}\right)+\mathrm{O}_{\ell_{\mathrm{i}}} \quad \mathrm{i} \in \mathrm{N}^{\mathrm{s}}
\end{aligned}
$$

In localization schema it is assumed that all nodes permanently move and the ordinary node uses an inertial system to self-estimate its speed and direction, which estimates $N$ positions $j^{\tilde{j}}=\left[\tilde{j}_{i}^{\sim}{ }_{i}^{\mathrm{x}}, \tilde{j}_{i}^{\sim}\right]$ of location $j_{i}$ at transmission time or reception time of ith packet in duration localization window. These locations will translate into a series of motion vectors $\omega_{\mathrm{i}, \mathrm{i}^{\prime}}=\left[\mathrm{d}_{\mathrm{i}, \mathrm{i}^{\prime}}, \psi_{\mathrm{i}, \mathrm{i}^{\prime}}\right], \mathrm{d}_{\mathrm{i}, \mathrm{i}^{\prime}}$ is the distance between two self-estimated locations $\tilde{j}_{\mathrm{i}}$ and $\tilde{j}_{\mathrm{i}^{\prime}}$, and $\psi_{\mathrm{i}, \mathrm{i}^{\prime}}$ is the angle between them. The elements of motion vector $\omega_{\mathrm{i}, \mathrm{i}^{\prime}}$ are

$$
\begin{gathered}
d_{i, i^{\prime}}^{\sim_{i}}=\left\|j_{i}-j_{i^{\prime}}\right\|_{2} i, i^{\prime} \in N \\
\tan \left(\psi_{i, i^{\prime}}\right)=j^{\sim y}{ }_{i}-j^{\sim y}{ }_{i^{\prime}} / j^{\sim x}{ }_{i}-j^{\sim x}{ }_{i^{\prime}}
\end{gathered}
$$

The self-estimated locations $\tilde{j}_{i}$, will not directly be used and errors accumulate with time, hence the accuracy of the motion vectors for all packet pairs $i$ , $i^{\prime}$ transmitted or received by the ordinary node during the localization window is considered. It is assumed that for $\mathrm{i}, \mathrm{i}^{\prime} \in \mathrm{N}$ the estimated distance $\mathrm{d}_{\mathrm{i}, \mathrm{i}^{\prime}}$ equals the real distance $\mathrm{d}_{\mathrm{i}, \mathrm{i}^{\prime}}$ and $\tilde{\psi}_{\mathrm{i}, \mathrm{i}^{\prime}}$ equals the real angle $\psi_{\mathrm{i}, \mathrm{i}^{\prime} \text {. }}$ 


\subsection{Step 1: Using TOA for initial locations}

At this phase, in order to calculate ordinary node's initial location, it is assumed that the ordinary node has been synchronized. This means that initial clock skew is assigned " 1 ", and initial clock offset is assigned " 0 ". Anchor node 1 is taken as the base node and therefore comparing with anchor node " 1 ", the time difference for anchor node " $e$ " is:

$$
\Delta t_{\ell 1}=t_{\ell}-t_{1}, 1=2, \ldots, \mathrm{L}
$$

where $t^{\wedge}{ }_{1}$ denotes the estimate of the TOA for base anchor node " 1 ", $t^{\wedge} \ell$ denotes the estimate of the TOA for base anchor node " $\ell$ ", and $\Delta t^{\wedge} \ell_{1}$ stands for the estimate of the TDOA for base anchor node "l". Therefore, the distance difference $d_{\ell_{1}}=d_{\ell}-d_{1}$ can be estimated as:

$$
d_{\ell_{1}}=\eta \Delta t_{\ell 1}^{\wedge}
$$

$\eta$ is the sound average propagation speed. Now the following matrices are defined:

$$
\begin{gathered}
\mathrm{M}=\left[\begin{array}{cc}
\mathrm{x}_{2} & \mathrm{y}_{2} \\
\mathrm{x}_{3} & \mathrm{y}_{3} \\
\vdots & \vdots \\
\mathrm{x}_{\mathrm{L}} & \mathrm{y}_{\mathrm{L}}
\end{array}\right] \quad \mathrm{D}=\left[\begin{array}{c}
-\hat{\mathrm{d}}_{21} \\
-\hat{\mathrm{d}}_{31} \\
\vdots \\
-\hat{\mathrm{d}}_{\mathrm{L} 1}
\end{array}\right] \\
\mathrm{Q}=1 / 2\left[\begin{array}{c}
\mathrm{x}_{2}{ }^{2}+\mathrm{y}_{2}{ }^{2}-\hat{\mathrm{d}}_{21} \\
\mathrm{x}_{3}{ }^{2}+\mathrm{y}_{3}{ }^{2}-\hat{\mathrm{d}}_{31} \\
\vdots \\
\mathrm{x}_{\mathrm{L}}{ }^{2}+\mathrm{y}_{\mathrm{L}}{ }^{2}-\hat{\mathrm{d}}_{\mathrm{L} 1}
\end{array}\right] \quad \mathrm{j}=\left[\begin{array}{l}
x_{r} \\
y_{r}
\end{array}\right]
\end{gathered}
$$

the least-squares method is used and the position of ordinary node can be estimated as:

$$
\mathrm{j}^{\wedge}=\mathrm{d}_{1} \mathrm{M}^{\mathrm{T}}+\mathrm{M}^{\mathrm{T}} \mathrm{Q}
$$

\subsection{Step 2: Quantization of Node Movements}

In this step, the locations of the ordinary node and the anchor nodes are quantized so that multiple ToA communication measurements are associated with the same pair of quantized locations. Consider the two packets $n, m, n \in N^{r}, m \in N^{s}$. If the two sets of ordinary node locations $\mathrm{j}_{\mathrm{n}}$ and $\mathrm{j}_{\mathrm{m}}$ and anchor node locations $\mathrm{p}_{\mathrm{n}}$ and $\mathrm{p}_{\mathrm{m}}\left(\ell_{\mathrm{n}}=\ell_{\mathrm{m}}=\ell\right)$ are associated with the same quantized locations $\mathrm{k}_{\rho}$ and $\mathrm{u}_{\ell, v}(\rho, v$ are used for count quantized locations) of the UL node and anchor node 1 , it is assumed that $\mathrm{T}^{\mathrm{pd}}{ }_{\mathrm{n}}=$ $\mathrm{T}_{\mathrm{m}}^{\mathrm{pd}}$ and Equations (2), (3) can be combined.

For quantize the locations of anchor nodes, subsets $\dot{U}_{\ell, v} \in \mathrm{N}$ are introduced that including all packets associated with the same anchor node $\ell$ so that for each pair of packets $n, m \in U_{\ell, v},\left\|p_{n}-p_{m}\right\|_{2}$ $<\Delta$ ( $\Delta$ is a fixed threshold). Next the location $\mathrm{p}_{\mathrm{i}}, \mathrm{i} \epsilon$ $\mathrm{U}_{1, v^{\prime}}$ associated with the quantized location $\mathrm{u}_{\ell, v}$. Correspondingly for quantize locations of the ordinary node, subsets of packets $\mathrm{K}_{\rho} \in \mathrm{N}$ so that for each pair of packets $n, m \in \mathrm{K}_{\rho}, \mathrm{d}_{\mathrm{n}, \mathrm{m}}<\Delta$ and associate location $\tilde{j}_{i}$, $i \in K_{\rho^{\prime}}$ with the quantized location $\mathrm{k}_{\rho}$. It is noteworthy that single packet can be associated with multiple subsets $\dot{U}_{\ell, v}$ and $K_{\rho}$.

There is a tradeoff for determining $\Delta$. If it is determined too large, the supposition of identical propagation delay is flawed, thus the accuracy of the synchronization is low. On the other hand if it is determined too small, there might not be enough two-way ToA measurements associated with each pair of quantized locations $\mathrm{k}_{\rho}$ and $\mathrm{u}_{\ell, v}$ and accuracy of the synchronization process is degraded.

\subsection{Step 3: Estimating the Clock Skews and Offsets}

In this phase the quantized locations is used to estimate clock skews $\mathrm{S}_{\ell}$ and offset $\mathrm{O}_{\ell}, 1=1,2,3, \ldots$ ., $\mathrm{L}$ and subsets $\mathrm{N}_{\ell}^{\mathrm{r}} \in \mathrm{N}$ and $\mathrm{N}_{\ell}^{\mathrm{s}} \in \mathrm{N}$ with cardinality $\underline{N}_{\ell}^{\mathrm{r}}$ and $\underline{\mathrm{N}}_{\ell}^{\mathrm{s}}$ including all packets associated with anchor node 1 are defined. The pair of packets $n \epsilon$ $\mathrm{N}_{\ell}^{\mathrm{r}}$ and $\mathrm{m} \in \mathrm{N}_{\ell}^{\mathrm{s}}$ is considered and locations $\mathrm{p}_{\mathrm{n}}$ and $\mathrm{p}_{\mathrm{m}}$ are mapped onto the same quantized locations $\mathrm{u}_{\ell, v^{\prime}}$ and locations $\tilde{j}_{\mathrm{n}}, \tilde{j}_{\mathrm{m}}$ are mapped onto the same quantized location $\mathrm{k}_{\rho}$. It is assumed that for each anchor node 1 , results are mapped into $M_{1}$ pairs of Equations (2) and (3) and $M_{\ell}$ increases with the quantization threshold $\Delta$. As said above, the differences between the propagation delays $\mathrm{T}^{\mathrm{pd}}{ }_{\mathrm{n}}$ and $\mathrm{T}_{\mathrm{m}}^{\mathrm{pd}}$ in Equations (2) and (3) are ignored, so obtain $\mathrm{M}_{\ell}$ equations:

$$
\begin{gathered}
\frac{\mathrm{R}_{\mathrm{n}}+\mathrm{T}_{\mathrm{m}}}{\mathrm{S}_{\mathrm{l}}}-\frac{2 \mathrm{O}_{\mathrm{l}}}{\mathrm{S}_{\mathrm{l}}}=\mathrm{T}_{\mathrm{n}}+\mathrm{R}_{\mathrm{m}}+\gamma_{\mathrm{n}}+\gamma_{\mathrm{m}} \\
, \mathrm{n} \in \mathrm{N}_{\ell}^{\mathrm{r}}, \mathrm{m} \in \mathrm{N}_{\ell}^{\mathrm{s}}
\end{gathered}
$$

$\mathrm{B}_{\ell}$ is an $\left[\mathrm{M}_{\ell} * 2\right]$ matrix with rows $\left[\mathrm{R}_{\mathrm{n}}+\mathrm{T}_{\mathrm{m}}-2\right]$. $b_{\ell}$ and $\epsilon_{\ell}$ are column vectors with elements $T_{n}+R_{m}$ and $\gamma_{\mathrm{n}}+\gamma_{\mathrm{m}}$. Now the LS estimator is applied

$$
\theta_{\ell}^{\wedge}=\left(\mathrm{B}_{\ell}^{\mathrm{T}} \mathrm{B}_{\ell}\right)^{-1} \mathrm{~B}_{\ell}^{\mathrm{T}} \mathrm{b}_{\ell}
$$

Using Equation (10) the covariance matrix of $\theta^{\wedge} \ell$ is calculated by the following relation [15]: 


$$
\mathrm{Q}_{\theta}=2 \sigma^{2}\left(\mathrm{~B}_{\ell}^{\mathrm{T}} \mathrm{B}_{\ell}\right)^{-1}
$$

The main diagonal elements of $\mathrm{Q}_{\theta}$ are proportional to $1 / \mathrm{M}_{\ell}$ and $1 / \mathrm{M}^{2}$, therefore for large $\mathrm{M}_{\ell}$, the estimates of $\theta^{\wedge}(1)$ and $\theta^{\wedge}(2)$ have much smaller variance than $\sigma^{2}$.

\subsection{Step 4: Stratification compensation}

After estimating clock skew and offset, the quantized locations are no longer in use and initial objective which is to estimate the propagation delay should be considered. Hence localization accuracy of the proposed method is not limited to $\Delta$. But as we know, in UWSNs due to the inhomogeneity of water medium in terms of the pressure, salinity, temperature, and etc., assuming the straight line transmission is wrong [16]. In this paper the method is adopted with the compensation of the above stratification effect.

It is assumed that the sound velocity profile (SVP) is only depth dependent. Let $\mathrm{v}(\mathrm{z})$ denotes the SVP as a function of depth $\mathrm{z}$, and $\left(\mathrm{y}_{\mathrm{s}}, \mathrm{z}_{\mathrm{s}}\right)$ and $\left(\mathrm{y}_{\mathrm{r}}, \mathrm{z}_{\mathrm{r}}\right)$ denote the location of the sender and the receiver. Furthermore, also the following assumptions are considered:

- The SVP, v (z) is available with extract from sea environmental variable.

- The depth of the receiver can be roughly estimated as $\mathrm{z}_{\mathrm{r}}$ using a depth-sensor.

The travel time for an acoustic ray along a possible path $\mathcal{S}$ is expressed as [16]

$$
T=\int_{\mathcal{S}} 1 / \mathrm{v}(s) \mathrm{d} s
$$

Define $y=f(z)$ and $f^{\prime}(z)=d y / d z$, so we have

$$
\mathrm{ds}=\sqrt{\mathrm{dz}^{2}+\mathrm{dy}^{2}}=\sqrt{1+\mathrm{f}^{\prime}(\mathrm{z})^{2}} \mathrm{~d}
$$

The travel time can be written as

$$
\mathrm{T}=\int_{\mathbf{z}_{\mathbf{s}}}^{\mathbf{z}_{\mathbf{r}}} \frac{\sqrt{1+\mathrm{f}^{\prime}(\mathrm{z})^{2}}}{\mathrm{v}(\mathrm{z})} \mathrm{dz}
$$

According to Fermat's principle [17], the true travel path is the one which minimizes the travel time; hence $\mathrm{f}(\mathrm{z})$ can be obtained via

$$
\frac{\mathrm{d}}{\mathrm{dz}} \frac{\partial}{\partial \mathrm{f},} \frac{\sqrt{1+\mathrm{f}^{\prime}(\mathrm{z})^{2}}}{\mathrm{v}(\mathrm{z})}=0
$$

This leads to

$$
\frac{f^{\prime}(z)}{v(z) \sqrt{1+f^{\prime}(z)^{2}}}=C
$$

where $\mathrm{C}$ is the integration constant. Therefore the travel path $\mathrm{f}(\mathrm{z})$ can be obtained from

$$
\mathrm{f}^{\prime}(\mathrm{z})=\frac{\mathrm{Cv}(\mathrm{Z})}{\sqrt{1-[\mathrm{Cv}(\mathrm{z})]^{2}}}
$$

Then $\hat{y_{r}}=\left(y_{r}-y_{s}\right)$, that is showed in Figure 2, can be obtained via the following formula [16]:

$$
\begin{gathered}
\hat{\mathrm{y}_{\mathrm{r}}}=\mathrm{f}\left(\mathrm{z}_{\mathrm{r}}\right)=\int_{z_{S}}^{z_{r}} f^{\prime}(z) d z= \\
\int_{z_{S}}^{z_{r}} \frac{\operatorname{Cv}(\mathrm{Z})}{\sqrt{1-[\operatorname{Cv}(\mathrm{z})]^{2}}} d z
\end{gathered}
$$

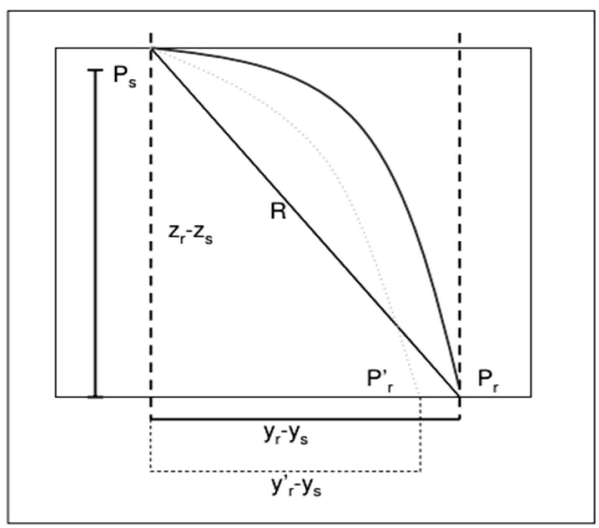

Fig. 2. Illustration of stratification effect [11]

Therefore, because, the initial location was estimated in Step 2, $\left(\mathrm{y}_{\mathrm{r}}-\mathrm{y}_{\mathrm{s}}\right)$ is known. Thus with Equation (18), by performing numerical search, a constant " $\mathrm{C}$ " can be obtained. Now by knowing "C", the propagation delay " $\mathrm{T}^{\mathrm{pd}}{ }_{\mathrm{i}}$ " can be estimated with considering stratification effect via

$$
\mathrm{T}_{\mathrm{i}}^{\wedge \mathrm{pd}}=\int_{\mathrm{Z}_{\mathrm{S}}}^{\mathrm{Z}_{\mathrm{r}}} \frac{1}{\mathrm{~V}(\mathrm{Z})} \frac{1}{\sqrt{1-[\mathrm{Cv}(\mathrm{z})]^{2}}} \mathrm{dz}
$$

\subsection{Step 5: Localization with Iterative Refinement}

In this step localization is performed using propagation delay estimations Equation (19). The 
objective of the localization step is to estimate the $\mathrm{j}^{\mathrm{x}}{ }_{\mathrm{N}}$ and $\mathrm{j}_{\mathrm{N}}^{\mathrm{y}}$ of ordinary node at the end of the localization window W. For this purpose, the common approach is adopted to linearize the estimation problem [18] and first the transformed variable vector $\varsigma_{N}=\left[\left(j^{x}\right)^{2}+\left(j^{y}\right)^{2}, j^{x}{ }_{N}, j^{y}{ }_{N}\right]^{T}$ is estimated.

Define

$$
\alpha_{\mathrm{i}, \mathrm{i}^{\prime}}=\frac{d_{\mathrm{i}, i^{\prime}}^{\tilde{x}^{\prime}}}{\sqrt{1+\tan \left(\psi_{\mathrm{i}, \mathrm{i}^{\prime}}\right)^{2}}}, \beta_{\mathrm{i}, \mathrm{i}^{\prime}}=\alpha_{\mathrm{i}, \mathrm{i}^{\prime}} \tan \left(\psi_{\mathrm{i}, \mathrm{i}^{\prime}}\right)
$$

It is assumed that $\mathrm{d}_{\mathrm{i}, \mathrm{i}^{\prime}}$ and $\tilde{\psi}_{\mathrm{i}, \mathrm{i}^{\prime}}$ in Equation (4) are equal to $\mathrm{d}_{\mathrm{i}, \mathrm{i}^{\prime}}$ and $\psi_{\mathrm{i}, \mathrm{i}^{\prime}}$ respectively (relying on the accuracy of the motion vectors during the localization window). Therefore:

$$
\mathrm{j}_{\mathrm{i}}^{\mathrm{x}}=\mathrm{j}_{\mathrm{i}}^{\mathrm{x}}-\alpha_{\mathrm{i}, \mathrm{i}^{\prime}}, \mathrm{j}_{\mathrm{i}^{\mathrm{i}}=\mathrm{j}_{\mathrm{i}}^{\mathrm{y}}-\beta_{\mathrm{i}, \mathrm{i}^{\prime}} \mathrm{i}, \mathrm{i}^{\prime} \in \mathrm{N}}
$$

Because of assuming small differences between $\mathrm{T}^{\wedge \mathrm{pd}}$ and actual $\mathrm{T}_{\mathrm{i}}^{\mathrm{pd}}$ the set of $\mathrm{N}$ equations are reduced to N-1 [12] in Equations (22)

$$
\begin{aligned}
& \mu_{\mathrm{N}, \mathrm{i}} \cdot \varsigma_{\mathrm{N}}=a_{\mathrm{N}, \mathrm{i}}+\epsilon_{\mathrm{N}, \mathrm{i}} \quad \mathrm{i}=1,2, \ldots, \mathrm{N}-1 \\
& \text { that } \mu_{\mathrm{N}, \mathrm{i}}=\left[\mu_{\mathrm{N}, \mathrm{i}}(1), \mu_{\mathrm{N}, \mathrm{i}}(2), \mu_{\mathrm{N}, \mathrm{i}}(3)\right] \text { and } \\
& a_{N, i}=\left(T^{\wedge p d}\right)^{2}\left(\left(p^{\mathrm{x}}\right)^{2}+\left(p^{\mathrm{y}}{ }_{\mathrm{N}}\right)^{2}\right) \\
& \text { - }\left(\mathrm{T}_{\mathrm{N}}^{\wedge \mathrm{pd}}\right)^{2}\left(\left(\mathrm{p}_{\mathrm{i}}^{\mathrm{x}}+\alpha_{\mathrm{N}, \mathrm{i}}\right)^{2}+\left(\mathrm{p}_{\mathrm{i}}^{\mathrm{y}}+\beta_{\mathrm{N}, \mathrm{i}}\right)^{2}\right) \\
& \mu_{\mathrm{N}, \mathrm{i}}(1)=\left(\mathrm{T}_{\mathrm{N}}^{\mathrm{pd}}\right)^{2}-\left(\mathrm{T}_{\mathrm{i}}^{\mathrm{Apd}}\right)^{2} \\
& \mu_{\mathrm{N}, \mathrm{i}}(2)=2\left(\mathrm{~T}^{\wedge \mathrm{pd}}{ }^{2}\right)^{2} \mathrm{p}_{\mathrm{N}}^{\mathrm{x}}-2\left(\mathrm{~T}^{\wedge \mathrm{pd}}{ }_{\mathrm{N}}\right)^{2}\left(\mathrm{p}_{\mathrm{i}}^{\mathrm{x}}+\alpha_{\mathrm{N}, \mathrm{i}}\right) \\
& \mu_{\mathrm{N}, \mathrm{i}}(3)=2\left(\mathrm{~T}_{\mathrm{i}}^{\wedge \mathrm{pd}}\right)^{2} \mathrm{p}^{\mathrm{y}}{ }_{\mathrm{N}}-2\left(\mathrm{~T}_{\mathrm{N}}^{\mathrm{pd}}\right)^{2}\left(\mathrm{p}_{\mathrm{i}}^{\mathrm{y}}+\beta_{\mathrm{N}, \mathrm{i}}\right)
\end{aligned}
$$

where $\epsilon_{\mathrm{N}, \mathrm{i}}$ is the noise that originate from the noisy estimations (19). For the localization window $\mathrm{W}$, an $[(\mathrm{N}-1) * 3]$ matrix A with rows $\mu_{\mathrm{N}, \mathrm{i}}$ and vectors a and $\epsilon$ with elements $a_{\mathrm{N}, \mathrm{i}}$ and $\epsilon_{\mathrm{N}, \mathrm{i}}$ are constructed. Afterwards (N-1) equations (22) are arranged in

$$
\mathrm{A}_{\varsigma_{\mathrm{N}}}=a+\epsilon
$$

The elements of the $\epsilon$ depend on the elements of $\varsigma_{\mathrm{N}}$, hence, direct estimation of $\varsigma_{\mathrm{N}}$ from Equation (24) produces result in low accuracy. Hence, the method in [18] will be followed and a two-step heuristic approach is offered in which first $\varsigma_{\mathrm{N}}$ is estimated, and then a refinement step is performed. The coarse estimate of $\varsigma_{\mathrm{N}}$ is given by

$$
\zeta^{\mathrm{LS}}{ }_{\mathrm{N}}=\left(\mathrm{A}^{\mathrm{T}} \mathrm{A}\right)^{-1} \mathrm{Aa}
$$

It is noteworthy that $\epsilon_{\mathrm{N}, \mathrm{i}}$ in Equation (22) can be formalized as $\gamma_{\mathrm{i}} \mathrm{f}_{\mathrm{N}, \mathrm{i}}$ where $\mathrm{f}_{\mathrm{Ni}}$ is a function of the elements of $\varsigma_{\mathrm{N}}$. hence $\epsilon_{\mathrm{N}, \mathrm{i}}$ are i.i.d random variables and the covariance matrix $\sigma^{2} \mathrm{Q}_{\mathrm{N}}$ of $\epsilon$ is a diagonal matrix that, whose ith diagonal element equals $\sigma^{2} f^{2}{ }_{N, i}$. with use the $\varsigma^{\mathrm{LS}}{ }_{\mathrm{N}}$ from Equation (25) to estimate the elements of $\mathrm{f}_{\mathrm{N}, \mathrm{i}}, \mathrm{i}=1,2,3, \ldots, \mathrm{N}-1, \mathrm{Q}_{\mathrm{N}}$ as $\mathrm{Q}_{\mathrm{N}}$ will be estimated. The purified estimate of $\varsigma_{\mathrm{N}}$ is derived as follows:

$$
\zeta^{\wedge \mathrm{WLS}}{ }_{\mathrm{N}}=\left(\mathrm{A}^{\mathrm{T}} \mathrm{Q}^{\wedge-1}{ }_{\mathrm{N}} \mathrm{A}\right)^{-1} \mathrm{~A} \mathrm{Q}^{\wedge-1}{ }_{\mathrm{N}} a
$$

where whose error covariance matrix is calcaulated based on the following formula [15] :

$$
Q^{\wedge^{\prime}}=\left(A^{T} Q^{\wedge-1}{ }_{N} A\right)^{-1}
$$

Finally, the inner connection of the elements of $\varsigma_{\mathrm{N}}$ is used to estimate the location vector $\mathrm{j}_{\mathrm{N}}$. Defining

$$
\mathrm{G}_{\mathrm{N}}=\left[\begin{array}{cc}
\zeta_{N}^{W L S}(2) & \zeta_{N}^{W L S}(3) \\
1 & 0 \\
0 & 1
\end{array}\right]
$$

$\varsigma^{\wedge \text { WLS }}(i)$ is ith element of $\varsigma^{\wedge L L}$. So we have

$$
\mathrm{G}_{\mathrm{N}} \mathrm{j}_{\mathrm{N}}=\varsigma_{\mathrm{N}}^{\mathrm{WLS}}+\epsilon_{\mathrm{N}}
$$

$\epsilon_{\mathrm{N}}$ is a [3*1] estimation noise of $\varsigma^{\mathrm{WLS}}{ }_{\mathrm{N}}$. using Equations (26), (27) and (28), the WLS estimator of $\mathrm{j}_{\mathrm{N}}$ is

$$
j^{\wedge}=\left(G^{T}{ }_{N} Q^{\wedge-1}{ }_{N} G_{N}\right)^{-1} G_{N} Q^{\wedge-1}{ }_{N} \zeta^{\text {WLS }}{ }_{N}
$$

The $\mathrm{j}_{\mathrm{N}}(1)=\mathrm{j}^{\wedge \mathrm{x}}$ and $\mathrm{j}_{\mathrm{N}}(2)=\mathrm{j}^{\wedge \mathrm{y}}$ that are the desired location coordinates.

The accuracy of estimation (29) depends on the quality of $\varsigma^{\wedge \mathrm{LS}}$ from (25) that used to construct the error covariance matrix, $\mathrm{Q}_{\mathrm{N}}$. Now [19] is followed and an iterative refinement procedure is proposed to improve the accuracy of $\mathrm{Q}_{\mathrm{N}}$.

In the kth step of iteration, vector $\hat{j}^{\wedge} \mathrm{N}, \mathrm{k}$ using (29) from which the vector $\varsigma_{\mathrm{N}, \mathrm{k}}$ is constructed and in the $(\mathrm{k}+1)$ step $\varsigma_{\mathrm{N}, \mathrm{k}}$ replaces $\varsigma^{\mathrm{NS}}{ }_{\mathrm{N}}$ in the construction of $\mathrm{Q}_{\mathrm{N}}$. As a stopping criterion, the covariance matrix of the kth estimation Equation (29) is used 


$$
\mathrm{Q}^{\wedge \prime \prime}, \mathrm{k}=\left(\mathrm{G}_{\mathrm{N}}^{\mathrm{T}} \mathrm{Q}^{\wedge,-1}{ }_{\mathrm{N}} \mathrm{G}_{\mathrm{N}}\right)^{-1}
$$

because the determinant, $\left|\mathrm{Q}^{\wedge \prime \prime} \mathrm{N,k}\right|$, is directly proportional to the estimation accuracy [15], the iteration stops is when the value of $\left|Q^{\wedge \prime \prime}, k\right|-\mid$ $\mathrm{Q}^{\wedge \prime}{ }_{\mathrm{N}, \mathrm{k}-1} \mid$ is below the threshold $\Delta_{\text {iter }}$ or if the number of iterations exceeds $\mathrm{N}_{\text {iter }}$.

\section{SIMULATION RESULTS}

The simulations are implemented in Matlab software and the experiments are performed with two anchor nodes and one ordinary node with communicating in a simple TDMA fashion. Anchor nodes and ordinary nod were placed uniformly in a square area of $1 * 1 \mathrm{~km}^{2}$ and moved between two near packet transmission times at uniformly distributed speed and angle between [-5, 5] knots unit of speed which equals one nautical mile per hour (6076 feet per hour) - and [0, 360] degrees, respectively. A zero mean independent and identically distributed (i.i.d.) Gaussian noise with variance $\sigma^{2}$ for each of the ToA estimations (see Equations (2) and (3)) is added. Moreover, considering the results in reference [20], a zero mean i.i.d. Gaussian noise with variance $1 \mathrm{~m}^{2}$ is added to each of the distance elements of the motion vectors (see Equation (4)). For simulating synchronization errors, the clock of each of nodes had a Gaussian distributed random skew and offset relative to a common clock with mean values 1 and $0 \mathrm{~s}$ and variances 0.001 and $0.5 \mathrm{~s}^{2}$, respectively.

Quantization threshold is adjusted $\Delta=38 \mathrm{~m}$ and a localization window is adjusted $\mathrm{W}=20$ time slots and time-slot duration is selected $\mathrm{T}_{\text {slot }}=5 \mathrm{~s}$, considering the long propagation delay in the WSNs channel (e.g., $4 \mathrm{~s}$ for a range of $6 \mathrm{~km}$ ). The MSEs of the time skew and time offset estimations are defined as $\mathrm{E}\left\{\left(\theta_{\mathrm{S}}-\theta_{\mathrm{S}}\right)^{2}\right\}$ and $\mathrm{E}\left\{\left(\theta_{\mathrm{O}}-\theta_{\mathrm{O}}\right)^{2}\right\}$ respectively.

Because STSL algorithm that is proposed in [12] and it was described in Related work section, is compared with previous methods, performance of the proposed method is compared with STSL algorithm. Hence, Equation (31) was used for calculating localization errors in STSL.

$$
\text { Perr }=\sqrt{E\left[\left(\hat{j_{N}^{x}}-j_{N}^{x}\right)^{2}+\left(\hat{j}_{N}^{y}-j_{N}^{y}\right)^{2}\right]}
$$

This will be used for comparing localization in the proposed method and STSL. For each situation that will be described below, experiment is repeated thirty times and the mean is calculated. These means are used for performance evaluation and comparison. In the following, simulation results will be discussed.

In Figure 3, Perr from Equation (31) as a function of $1 / \sigma^{2}$ is shown. It is observed that error for the proposed method decreases with $1 / \sigma^{2}$, Hence, this method compensates for synchronization uncertainties and this method achieves higher accuracy than STSL algorithm in same situation. This is because it does not assume the straight line acoustic transmission.

Figure 4 and Figure 5 show the results of clock offset and clock skew estimations, illustrating that the proposed method has higher accuracy than STSL algorithm because of considering that sound waves don't propagate in straight line and sound propagation speed varies with depth.

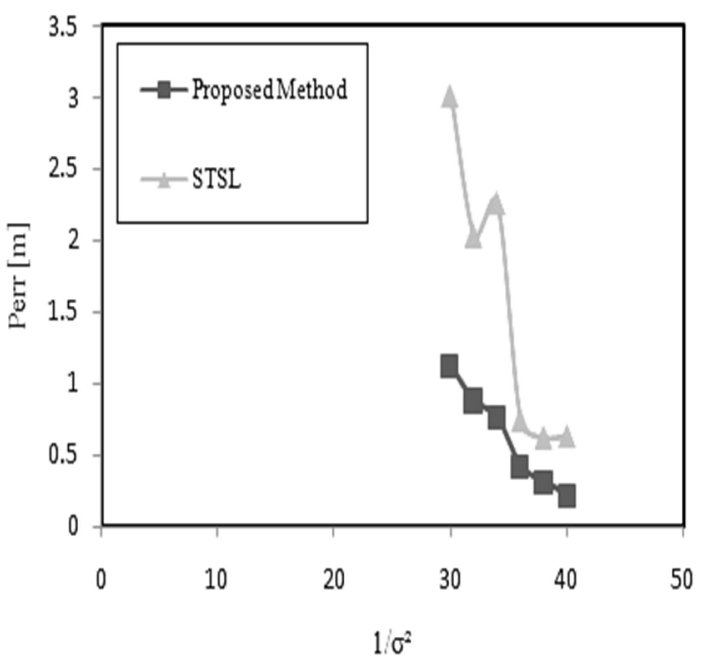

Fig. 3. Perr from Eq. (31) for TOA detection error variance $1 / \sigma^{2}$

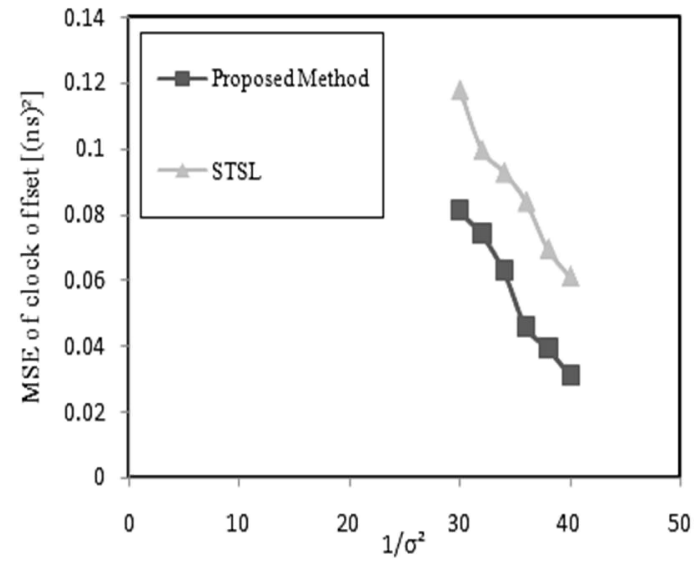

Fig. 4. MSE of clock offset versus TOA detection error variance $1 / \sigma^{2}$ 


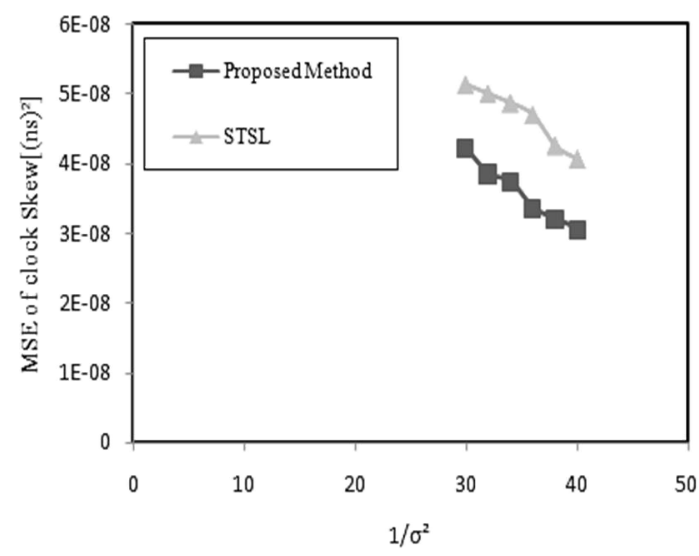

Fig. 5. MSE of clock Skew versus TOA detection error variance $1 / \sigma^{2}$

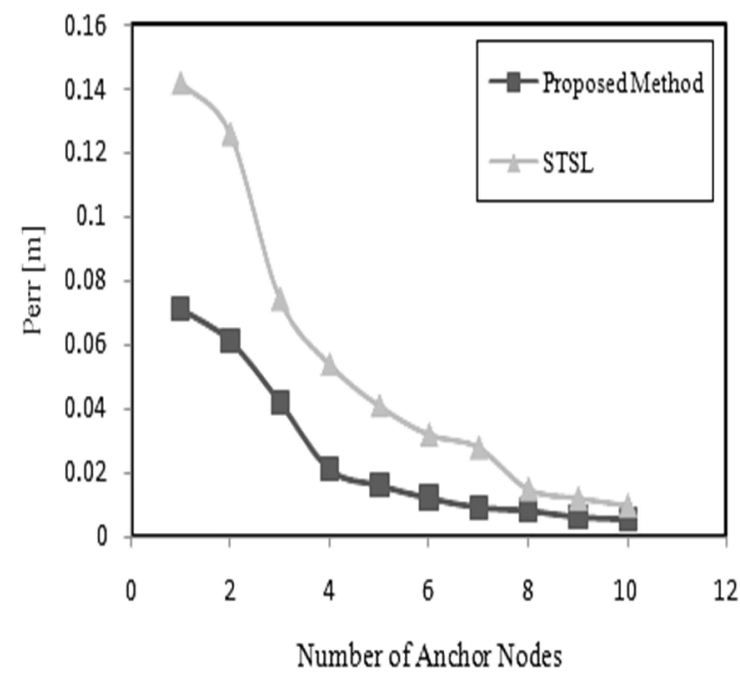

Fig. 6. Perr from Eq. (31) vs number of anchor node, with TOA detection error variance $1 / \sigma^{2}=45 \mathrm{db}$

In Figure 6, relation between Perr and number of anchor nodes is shown. Results show that with more anchor nodes, the results become better. This is because when more anchor nodes are involved, more data can be collected in the initial position estimations and synchronization procedure. Both of these will help to improve localization accuracy. With comparing by STSL, results also show that the proposed method with fewer number of anchor nodes achieve better accuracy.

\section{CONCLUSION}

In this paper, a new method was proposed that jointly solves the synchronization and localization problems in UWSNs which compensates the stratification effect in this type of networks. This method utilizes the constant movements of nodes and relies on packet exchange to acquire multiple ToA measurements at different locations.

Simulations results demonstrated that this method can cope with synchronization uncertainties in a dynamic environment, and attains reasonable localization accuracy.

\section{REFERENCES}

[1] P. Xie, L. Lao, and J.-H. Cui, "VBF: vectorbased forwarding protocol for underwater sensor networks", in To appear in Proceedings of IFIP Networking, May 2006.

[2] H. Yan, Z. Shi, and J.-H. Cui, "DBR: depthbased routing for underwater sensor networks", Proceedings of IFIP Networking, May 2008.

[3] M. Erol, H. Mouftah, and S. Oktug, "Localization Techniques for Underwater Acoustic Sensor Networks", IEEE Comm. Magazine, vol. 48, June, 2010, pp. 152-158.

[4] X. Cheng, H. Shu, Q. Liang, and D. Due, "Silent Positioning in Underwater Acoustic Sensor Networks", IEEE Trans. Vehicular Technology,, vol. vol. 57, no. 3, May, 2008, pp. 1756-1766.

[5] H. Tan, A. Eu, and W. Seah, "An Enhanced Underwater Positioning System to Support Deepwater Installations", Proc. IEEE Int'1 Conf. Comm. (ICC), vol. vol. 15, no. 2, June, 2005, pp. 88-95.

[6] H. Tan, A. Gabor, Z. Eu, and W. Seah, "A Wide Convergence Positioning System for Underwater Localization", Proc. IEEE Int'l Conf. Comm. (ICC), Mar, 2010.

[7] D. Mirza, and C. Schurgers, "Motion-aware self-localization for under-water networks", Third ACM Intl Workshop Wireless Network Testbeds, Experimental Evaluation and Characterization (WuWNeT 08), vol. 57, no. 3 , pp. 51-58.

[8] E. Kim, S. Lee, C. Kim, and K. Kim, "Floating Beacon-Assisted 3-D Localization for Variable Sound Speed in Underwater Sensor Networks", Proc. IEEE Sensors Conf, Nov, 2010, pp. 682685.

[9] M. Isik, and O. Akan, "A Three Dimentional Localization Algorithm for Underwater Acoustic Sensor Networks", IEEE Trans. Wireless Comm, vol. vol. 8, no. 9, Sept, 2009, pp. 4457-4463.

[10]C. Tian, W. Liu, J. Jin, Y. Wang, and Y. Mo, "Localization and Synchronization for 3D Underwater Acoustic Sensor Networks", Ubiquitous Intelligence and Computing, Lecture Notes in Computer Science J. 
Indulska, J. Ma, L. Yang, T. Ungerer and J. Cao, eds, Springer Berlin Heidelberg , 2007, pp. 622-631.

[11] J. Liu, Z. Wang, Z. M, Z. Peng, J.-H. Cui, and S. Zhou, "JSL: Joint time synchronization and localization design with stratification compensation in mobile underwater sensor networks", Sensor, Mesh and Ad Hoc Communications and Networks (SECON), 9th Annual IEEE Communications Society Conference on, 2012, pp. 317-325.

[12]R. Diamant, and L. Lampe, "Underwater Localization with Time-Synchronization and Propagation Speed Uncertainties", Mobile Computing, IEEE Transactions on, vol. 12, no. 7, 2013 ,pp. 1257-1269.

[13]A. Syed, and J. Heidemann, "Time Synchronization for High Latency Acoustic Networks", Proc. IEEE INFOCOM, Apr, 2006.

[14] K. Noh, Q. M. Chaudhari, E. Serpedin, and B. W. Suter, "Novel clock phase offset and skew estimation using two-way timing message exchanges for wireless sensor networks", IEEE Trans. Commun, vol. 55, no. 4, 2007, pp. 766777.

[15] S. Kay, Fundamentals of Statistical Signal Processing: Estimation Theory, Prentice-Hall, 1993.

[16] C. R. Berger, S. Zhou, P. Willett, and L. Liu, "Stratification effect com-pensation for improved underwater acoustic ranging", IEEE Transactions on Signal Processing, vol. 56, no. 8, 2008, pp. 539-546.

[17]E. W. Weisstein, "Fermat's Principle", World of Science-A Wolfram Web Resource [Online]. http://scienceworld.wolfram.com/ physics/FermatsPrinciple.html.

[18] J. Zheng, and Y. Wu, "Joint Time Synchronization and Localization of an Unknown Node in Wireless Sensor Networks", IEEE Trans. Signal Processing, vol. vol. 58, no. 3, Mar, 2010.

[19]W. Foy, "Position-Location Solutions by Taylor-Series Estima-tion”, IEEE Trans. Aerospace Electronic Systems, vol. vol. 12, no. 2, Mar, 1975, pp. 187-194.

[20]C. Lee, P. Lee, S. Hong, and S. Kim, "Underwater Navigation System Based on Inertial Sensor and Doppler Velocity Log Using Indirect Feedback Kalman Filter",
Offshore and Polar Eng, vol. vol. 15, no. 2, June, 2005, pp. 88-95.

\section{APPENDIX}

Table 1: List of notations

\begin{tabular}{|c|c|}
\hline Notation & Explanation \\
\hline $\mathrm{L}$ & $\begin{array}{c}\text { Number of anchor nodes } \\
\text { directly connected to ordinary } \\
\text { nod }\end{array}$ \\
\hline $\mathrm{j}_{\mathrm{i}}$ & $\begin{array}{c}\text { 2-D UTM coordinates of the } \\
\text { ordinary node at the time it } \\
\text { transmit or receives the ith } \\
\text { packet }\end{array}$ \\
\hline $\mathrm{t}_{1}$ & Local clock of anchor node 1 \\
\hline$\overline{p_{i}}$ & $\begin{array}{c}\text { 2-D UTM coordinates of the } \\
\text { th anchor node at the time it } \\
\text { transmit or receives the ith } \\
\text { packet }\end{array}$ \\
\hline $\mathrm{C}$ & Sound speed in water $[\mathrm{m} / \mathrm{sec}]$ \\
\hline $\mathrm{W}$ & $\begin{array}{c}\text { Duration of localization } \\
\text { window }\end{array}$ \\
\hline $\mathrm{N}$ & $\begin{array}{c}\text { Number of packet transmitted } \\
\text { during localization window } \\
\text { between ordinary node and } \\
\text { anchor nodes during the } \\
\text { localization window }\end{array}$ \\
\hline$\overline{S_{1}}$ & $\begin{array}{c}\text { Clock skew of the ordinary } \\
\text { node relative to the lth anchor } \\
\text { node }\end{array}$ \\
\hline $\mathrm{O}_{1}$ & $\begin{array}{l}\text { Clock offcet of the ordinary } \\
\text { node relative to the lth anchor } \\
\text { node [sec] }\end{array}$ \\
\hline $\mathrm{T}^{\mathrm{pd}}{ }_{\mathrm{i}}$ & $\begin{array}{c}\text { Propagation delay of the ith } \\
\text { packet [sec] }\end{array}$ \\
\hline $\mathrm{T}_{\mathrm{i}}$ & $\begin{array}{l}\text { Transmission local time of the } \\
\text { ith packet [sec] }\end{array}$ \\
\hline$\overline{\mathrm{R}_{\mathrm{i}}}$ & $\begin{array}{l}\text { Reception local time of the ith } \\
\text { packet [sec] }\end{array}$ \\
\hline $\mathrm{d}_{\mathrm{i}, \mathrm{i}^{\prime}}$ & $\begin{array}{l}\text { Self estimation of distance } \\
\text { between location } \mathrm{j}_{\mathrm{i}} \text { and } \mathrm{j}_{\mathrm{i}^{\prime}}\end{array}$ \\
\hline${\tilde{\psi}{\tilde{\mathrm{i}}, \mathrm{i}^{\prime}}^{\prime}}$ & $\begin{array}{l}\text { Self estimation of angle } \\
\text { between locations } \mathrm{j}_{\mathrm{i}} \text { and } \mathrm{j}_{\mathrm{i}^{\prime}} \\
{[\mathrm{rad}]}\end{array}$ \\
\hline$\Delta$ & $\begin{array}{c}\text { Threshold for location } \\
\text { quantization [m] }\end{array}$ \\
\hline$\sigma^{2}$ & $\begin{array}{c}\text { Variance of TOA } \\
\text { measurement noise }\left[\mathrm{sec}^{2}\right]\end{array}$ \\
\hline$\eta$ & $\begin{array}{c}\text { the sound average propagation } \\
\text { speed }\end{array}$ \\
\hline $\mathrm{L}$ & $\begin{array}{c}\text { Number of anchor nodes } \\
\text { directly connected to ordinary } \\
\text { nod }\end{array}$ \\
\hline
\end{tabular}

